\title{
INFLUÊNCIA DA TEMPERATURA NA FECUNDIDADE E LONGEVIDADE DE ADULTOS DE Diabrotica speciosa
}

\author{
Crébio José Ávila ${ }^{1}$ \\ José Roberto P. Parra²
}

\section{RESUMO}

O trabalho teve como objetivo determinar o efeito da temperatura sobre a fecundidade e a longevidade de adultos de Diabrotica speciosa, quando criados e mantidos em laboratório, em três temperaturas $(20,25$ e $30^{\circ} \mathrm{C}$ ). Os períodos de pré-oviposição (PPO) e de oviposição (PO), bem como a fecundidade e a longevidade de adultos (machos e fêmeas) de $D$. speciosa foram significativamente influenciados pelos regimes de temperaturas estudados. O PPO foi maior a $20^{\circ} \mathrm{C}$ que a 25 e $30^{\circ} \mathrm{C}$ sem, no entanto, diferir entre estas duas últimas temperaturas. O PO decresceu significativamente com o aumento da temperatura, o qual variou, em média, de 68,9 dias $\left(20^{\circ} \mathrm{C}\right)$ para 9,8 dias $\left(30^{\circ} \mathrm{C}\right)$. Efeito semelhante, foi observado em relação à longevidade de adultos, quando os mesmos mantiveram-se vivos, em média, mais de 100 dias na temperatura de $20^{\circ} \mathrm{C}$ e menos de 21 dias a $30^{\circ} \mathrm{C}$. $\mathrm{O}$ número total de ovos postos por fềmea foi semelhante para os insetos criados e mantidos nas temperaturas de 20 e $25^{\circ} \mathrm{C}$ e significativamente menor na temperatura de $30^{\circ} \mathrm{C}$. Com base nos resultados obtidos, conclui-se que a fecundidade e a sobrevivência de adultos de $D$. speciosa é influenciada pelas condições térmicas em que o inseto é criado e mantido na fase adulta, sendo a temperatura de $30^{\circ} \mathrm{C}$ menos adequada para a reprodução desta espécie, quando comparada às de $20 \mathrm{e} 25^{\circ} \mathrm{C}$.

Palavras-chave: vaquinha, temperatura, fecundidade, e longevidade de adultos.

1 Embrapa Agropecuaria Oeste, Caixa Postal 661, 79.804-970 - Dourados, MS. Email: crebio@cpao.embrapa.br, Brasil

2 ESALQ-USP, Dep. Entomol., Fitopatologia e Zoologia Agrícola, 13418-900 Piracicaba, SP. 


\section{ABSTRACT}

\section{INFLUENCE OF TEMPERATURE ON FECUNDITY AND ADULT LONGEVITY OF Diabrotica speciosa}

The aim of this work was to determine the fecundity and adult longevity of Diabrotica speciosa when reared and kept at the adult phase under laboratory conditions at three temperatures $\left(20,25\right.$ and $\left.30^{\circ} \mathrm{C}\right)$. The pre-oviposition and oviposition periods and the adult longevity (males and females) of $D$. speciosa were significantly influenced by the thermal conditions on which the insects were reared and kept. The pre-oviposition period was longer at $20^{\circ} \mathrm{C}$ than at 25 and $30^{\circ} \mathrm{C}$. There was no significant difference between the two latter temperatures. The oviposition period decreased significantly with the increase of temperature from 68,9 days $\left(20^{\circ} \mathrm{C}\right)$ to 9,8 days $\left(30^{\circ} \mathrm{C}\right)$. Similar effect was observed in relation to adult longevity. Males or females stayed alive more than 100 days at 20 ${ }^{\circ} \mathrm{C}$ and less than 21 days at $30^{\circ} \mathrm{C}$. The total number of eggs laid by the females was similar for the insects reared and kept under the temperatures of 20 and $25^{\circ} \mathrm{C}$ and significantly smaller at $30^{\circ} \mathrm{C}$. Based on these results, it could be verified that the fecundity and the adult longevity of $D$. speciosa are influenced by the thermal conditions under which the insect is reared. The $30^{\circ} \mathrm{C}$ temperature is less appropriate than 20 and $25^{\circ} \mathrm{C}$ for rearing this species.

Key words: Corn rootworm, temperature, fecundity, adult longevity.

\section{INTRODUÇÃO}

Diabrotica speciosa (Germar, 1824) (Coleoptera: Chrysomelidae) é uma espécie que ocorre em, praticamente, todos os Estados brasileiros, bem como em alguns países da América do Sul (Aréstegui, 1976; Krysan, 1986; Bercellini \& Malacalza, 1994). O adulto alimenta-se da parte aérea de várias plantas, enquanto as larvas, que são de hábito subterrâneo, zausam danos em raízes e tubérculos (Gassen, 1989). 
A temperatura é um dos fatores climáticos que mais afeta a biologia e o comportamento dos insetos, podendo influenciar a taxa de desenvolvimento e a viabilidade de suas fases imaturas, bem como a longevidade e a reprodução em adultos. A influência da temperatura, na faixa de 18 a $32^{\circ} \mathrm{C}$, sobre a duração e viabilidade das fases de ovo, larva e pupa de $D$. speciosa foi determinada por Milanez \& Parra (2000). No entanto, pesquisas visando avaliar a influência desse fator ambiental sobre adulto dessa praga, ainda não foram realizadas. Este trabalho teve como objetivo determinar o efeito da temperatura sobre fecundidade e longevidade de adultos de $D$. speciosa, quando criados e mantidos sob três condições térmicas, no laboratório.

\section{MATERIAL E MÉTODOS}

A pesquisa foi conduzida no Laboratório de Biologia de Insetos do Departamento de Entomologia, Fitopatologia e Zoologia Agrícola da Escola Superior de Agricultura Luiz de Queiroz (ESALQ). D. speciosa foi criada em condições de laboratório, segundo metodologia descrita por Ávila et al. (2000), nas temperaturas de 20,25 e $30^{\circ} \mathrm{C}$. Adultos recém-emergidos nessas três condições térmicas foram sexados, colocando-se um casal por gaiola cônica de acrílico transparente $(13,0 \mathrm{~cm}$ de altura por 8,5 e $6,0 \mathrm{~cm}$ de diâmetros de base e topo, respectivamente), que continha pequenas aberturas no topo, vedadas com o tecido "voil" para permitir aeração. No interior da gaiola, folíolos de feijoeiro foram mantidos em pequenos vidros com água para alimentação dos insetos, e também colocado um recipiente de plástico medindo $3,0 \mathrm{~cm}$ de diâmetro por $1,5 \mathrm{~cm}$ de altura, contendo gaze umedecida de cor preta, utilizada como substrato para oviposição (Milanez, 1995). As gaiolas foram mantidas em câmaras climatizadas do tipo BOD, nas mesmas condições de temperatura, umidade relativa e fotofase em que os insetos foram criados, utilizando-se vinte casais por temperatura. $\mathrm{O}$ alimento foi trocado a cada quatro dias, ocasião em que também se efetuava a contagem de ovos no substrato de oviposição de cada gaiola. Foram também determinados os períodos de pré-oviposição e de oviposição, bem como as 
longevidades de machos e de fêmeas, nas três condições térmicas.

O ensaio, inteiramente casualizado, teve os tratamentos constituidos pelas três temperaturas $\left(20,25\right.$ e $30^{\circ} \mathrm{C}$ ), em 20 repetições (gaiola com um casal). As variáveis avaliadas foram submetidas à análise de variância e as médias, comparadas pelo teste de Tukey, ao nível de $5 \%$ de probabilidade.

\section{RESULTADOS E DISCUSSÃO}

Os períodos de pré-oviposição (PPO) e de oviposição (PO), a longevidade de machos e de fêmeas, bem como a fecundidade de $D$. speciosa, foram significativamente influenciados pelos regimes de temperaturas sob os quais os insetos foram criados e mantidos durante a fase adulta (Tabela 1 e 2). O PPO a $20^{\circ} \mathrm{C}$ foi superior aos observados a 25 e $30^{\circ} \mathrm{C}$, mas não diferiu entre estas duas últimas temperaturas. O PO decresceu significativamente com o aumento da temperatura, que variou, em média, de $68,9\left(20^{\circ} \mathrm{C}\right)$ para 9,8 dias $\left(30^{\circ} \mathrm{C}\right)$. Efeito semelhante foi observado em relação à longevidade de machos e fềmeas, sendo que os mesmos mantiveram-se vivos, em média, mais de 100 dias na temperatura de $20^{\circ} \mathrm{C}$ e menos de 21 dias a $30^{\circ} \mathrm{C}$ (Tabela 1). Resultados semelhantes, com relação aos períodos de pré-oviposição, oviposição e longevidade, foram observados por Naranjo \& Sawyer (1987) quando casais da espécie $D$. barberi foram mantidos em sete diferentes temperaturas constantes, na faixa térmica de 15 a $30^{\circ} \mathrm{C}$. Elliott et al. (1990) também verificaram que a longevidade de adultos de $D$. virgifera virgifera decresceu com aumento de temperatura, no intervalo de 16 a $30^{\circ} \mathrm{C}$.

$\mathrm{O}$ número total de ovos postos por fêmea de $D$. speciosa foi semelhante nas temperaturas de 20 e $25^{\circ} \mathrm{C}$ e, significativamente menor na temperatura de $30^{\circ} \mathrm{C}$ (Tabela 2 ). Estes resultados discordam dos obtidos por Naranjo \& Sawyer (1987) os quais verificaram maior fecundidade de $D$. barberi a $25^{\circ} \mathrm{C}$ do que a $20^{\circ} \mathrm{C}$, embora se trate de espécies diferentes. Por outro lado, esses autores constataram redução significativa da capacidade de postura desta espécie, quando os adultos foram mantidos na temperatura de $30^{\circ} \mathrm{C}$, à semelhança do observado neste trabalho com $D$. speciosa. 
Tabela 1. Períodos de pré-oviposição, oviposição e longevidade de adultos (machos e fêmeas) de Diabrotica speciosa, mantidos com dieta natural (feijoeiro) sob três regimes de temperaturas. UR: $60 \pm 10 \%$, fotofase: 14 horas.

\section{Temperatura}

$\left({ }^{\circ} \mathrm{C}\right)$

20

25

30
Periodo (dias)

Pré-oviposição

$13,3 \pm 1,30 \mathrm{a}$

$8,5 \pm 0,68 b$

$8,1 \pm 0,71 b$

\section{Oviposição ${ }^{1}$}

$68,9 \pm 6,12 a$

$33,3 \pm 2,75 b$

$9,8 \pm 1,39 \mathrm{c}$
Longevidade (dias)

Macho

Fêmea

Médias seguidas da mesma letra, não diferem estatisticamente entre si, pelo teste de Tukey, ao nível de $5 \%$ de probabilidade.

Tabela 2. Número médio de ovos por fêmea de Diabrotica speciosa, mantidas com dieta natural (feijoeiro), sob três regimes de temperaturas. UR: $60 \pm 10 \%$, fotofase: 14 horas.

Temperatura $\left({ }^{\circ} \mathrm{C}\right)$

20

25

30

\section{$N^{\circ}$ de ovos/fêmea}

$799,5 \pm 127,6 \mathrm{a}$

$833,9 \pm 86,2$ a

$304,9 \pm 68,7 b$

Médias seguidas da mesma letra, não diferem estatisticamente entre si, pelo teste de Tukey, ao nivel de $5 \%$ de probabilidade.

Uma maior taxa metabólica do inseto, quando mantido a $30^{\circ} \mathrm{C}$, poderia ser uma das causas desta inadequação, uma vez que Gates, citado por Mattson \& Scriber (1987), afirmou que a taxa respiratória de animais poiquilotérmicos aumenta de 1,3 a 4,0 vezes, a cada incremento de $10{ }^{\circ} \mathrm{C}$ da temperatura ambiente em que se encontram. Assim, a energia 
acumulada pelo inseto através da alimentação foi, provavelmente, dissipada pela respiração ou por outras possíveis rotas metabólicas energéticas do inseto, o que afetou negativamente sua fecundidade e sobrevivência. Estudos nesta linha de pesquisa, poderão contribuir para o entendimento dos fatores que regulam a distribuição e a dinâmica populacional de $D$. speciosa nos agroecossistemas.

Com base nos resultados obtidos, conclui-se que a fecundidade e a sobrevivência de adultos $D$. speciosa são influenciados pelas condições térmicas em que o inseto é criado e mantido na fase adulta, sendo a temperatura de $30{ }^{\circ} \mathrm{C}$ menos adequada para a reprodução desta espécie, quando comparada às de 20 e $25^{\circ} \mathrm{C}$. Embora o número de ovos obtidos a $20^{\circ} \mathrm{C}$ não diferisse daquele a $25^{\circ} \mathrm{C}$ (Tabela 2), o período de oviposição observado na primeira condição térmica foi mais que o dobro na segunda temperatura. Com base nesse parâmetro, pode-se concluir que a temperatura de $25^{\circ} \mathrm{C}$ é a mais adequada para a criação de $D$. speciosa, em condições de laboratório.

\section{REFERÊNCIAS BIBLIOGRÁFICAS}

ARÉSTEGUI, A.P., 1976. Plagas de la Papa en Andahuaylas-Apurimac. Revista Peruana de Entomologia, 19(1):97-98.

ÁVILA C.J.; A.C.P. TABAI \& J.R.P. PARRA, 2000. Comparação de Técnicas para Criação de Diabrotica speciosa (Germar) (Coleoptera: Chrysomelidae) em Dietas Natural e Artificial. Anais da Sociedade Entomológica do Brasil, 29(2):257-267.

3ERCELLINI, N. \& L. MALACALZA, 1994. Plagas y Predadores en

Soja en el Noroeste de la Provincia de Buenos Aires (Arg.). Turrialba, 44(4):244-254.

ILLIOTT, N.C.; D.R. LANCE \& S.L. HANSON, 1990. Quantitative

Description of the ilnfluence of Fluctuating Temperatures on the

Reproductive Biology and Survival of the Western corn Rootworm,

Diabrotica virgifera virgifera LeConte (Coleoptera: Chrysomelidae).

Canadian Entomologist, 122:59-68. ¡ASSEN, D.N., 1989. Insetos Subterrâneos Prejudiciais às Culturas 
no Sul do Brasil. Passo Fundo: EMBRAPA-CNPT, 49p. (EMBRAPA-CNPT, Documento, 13).

KRYSAN, J.L., 1986. Introduction: Biology, Distribution, and Identification of Pest Diabrotica. In: KRYSAN, J.L.; MILLER, T.A. (Ed.) Methods for Study of Pest Diabrotica. New York: Springer Verlog, cap.1, p.1-23.

MATTSON, W.J. \& J.M. SCRIBER, 1987. Nutritional Ecology of Insect

Folivores of Wood Plants: Nitrogen, Water, Fiber, and Mineral Considerations. In: SLANSKY Jr., F.; RODRIGUEZ, J.G. (Ed.). Nutritional Ecology of Insects, Mites, Spiders, and Related Invertebrates. New York: John Wiley, cap.3, p.105-143.

MILANEZ, J.M., 1995. Técnicas de Criação e Bioecologia de Diabrotica speciosa (Germar, 1824) (Coleoptera: Chrysomelidae). Piracicaba, 102p. Tese (Doutorado) - Escola Superior de Agricultura "Luiz de Queiroz", Universidade de São Paulo.

MILANEZ, J.M. \& J.R.P. PARRA, 2000. Biologia e Exigências Térmicas de Diabrotica speciosa (Germar) (Coleoptera: Chrysomelidae) em Laboratório. Anais da Sociedade Entomológica do Brasil, 29(1):23-29.

NARANJO, S.E. \& A.J. SAWYER, 1987. Reproductive Biology and Survival of Diabrotica barberi (Coleoptera: Chrysomelidae): Effect of Temperature, Food, and Seasonal Time of Emergence. Annals of the Entomological Society of America, 80(6):841-848. 